\title{
Impact of early malnourishment on the chronic inflammatory response and its implications for the effect of indomethacin on Wistar rats
}

\author{
Thiago de Oliveira Assis ${ }^{1}$, Teresinha Gonçalves da Silva ${ }^{2}$, Eryvelton de Souza Franco ${ }^{3}$, Ana Catarina \\ Rezende Leite ${ }^{3}$, Silvia Regina Arruda de Moraes $^{4}$ and Maria Bernadete de Sousa Maia ${ }^{3 *}$ \\ ${ }^{1}$ Department of Morphology, Federal University of Paraíba, Cidade Universitária, CEP 58051-900, João Pessoa, PB, Brazil \\ ${ }^{2}$ Department of Antibiotics, Federal University of Pernambuco, Avenida Professor Moraes Rego, s/n, Cidade Universitária, \\ 52670-901 Recife, PE, Brazil \\ ${ }^{3}$ Department of Physiology and Pharmacology, Laboratory of Pharmacology of Bioactive Products, Federal University of \\ Pernambuco, Avenida Professor Moraes Rego, s/n, Cidade Universitária, 52670-901 Recife, PE, Brazil \\ ${ }^{4}$ Department of Anatomy, Federal University of Pernambuco, Avenida Professor Moraes Rego, s/ $n$, Cidade Universitária, \\ 52670-901 Recife, PE, Brazil \\ (Received 19 May 2010 - Revised 30 December 2010 - Accepted 9 February 2011 - First published online 9 May 2011)
}

\section{Abstract}

The objective of the present study was to investigate whether early undernutrition changes the chronic inflammatory response, so as to study its influence on pharmacological response to indomethacin. Rat offspring of dams fed from the first day of gestation to term or throughout the lactation period received a balanced diet (NN) or a basic regional diet (BRD) from northeast Brazil. According to their dams, the offspring were divided into three groups: NN; basic regional diet during gestation (BRD-g, undernourished during gestation); basic regional diet during gestation and lactation (BRD-gl, undernourished during gestation and lactation). At 2 months of age, Freund's adjuvant $(0.2 \mathrm{ml})$ was inoculated into the plantar surface of the hind paw (day 0) of animals. All animals orally received saline $(0.9 \%)$ for $28 \mathrm{~d}$. Another group of adult offspring was subjected to the same procedure as described above, but orally received indomethacin $(2 \mathrm{mg} / \mathrm{kg})$ instead of saline, and divided into three subgroups: NN treated with indomethacin (NNI); BRD-g treated with indomethacin (BRDI-g); BRD-gl treated with indomethacin (BRDI-gl). The hind paw volume was calculated on days 0 (initial paw volume), 7, 14 and 28. Hind paw swelling, blood albumin and C-reactive protein (CRP) levels and leucocyte counts were evaluated as markers of inflammation. Reduced hind paw swelling and the blood levels of serum albumin and CRP were found in the BRD-g and BRD-gl offspring. However, no difference was found in the leucocyte count. Compared with their respective saline-treated groups (NN, BRD-g and BRD-gl), the anti-inflammatory effect of indomethacin was lower in the BRDI-g and BRDI-gl groups than in the NNI group. We conclude that early undernutrition attenuated the chronic inflammatory response and the anti-inflammatory effect of indomethacin.

Key words: Undernutrition: Chronic inflammation: Indomethacin: Pregnancy

The growing number of individuals with nutritional deficiency is an emerging global problem that poses significant economic and public health consequences ${ }^{(1)}$. Epidemiological findings show that maternal undernutrition affects fetal development and predisposes the offspring to an increased incidence of diseases such as hypertension, type 2 diabetes and $\mathrm{CHD}^{(2-4)}$ as well as leucocyte reduction at the inflammatory site ${ }^{(5)}$. Concerning the inflammatory response, experimental findings indicate that malnutrition impairs the acute ${ }^{(6)}$ and systemic inflammatory responses ${ }^{(7-9)}$.

The systemic inflammatory response represents a coordinated set of physiological events to fight against infections and many other injuries to which the organism is exposed ${ }^{(10,11)}$. In a healthy individual, the inflammatory response to tissue lesion or infection is fast and efficient, with specific resolution occurring even before the involvement of the immune

Abbreviations: BRD, basic regional diet; BRD-g, basic regional diet during gestation; BRD-gl, basic regional diet during gestation and lactation; BRDI-g, basic regional diet treated with indomethacin during gestation; BRDI-gl, basic regional diet treated with indomethacin during gestation and lactation; CRP, C-reactive protein; FCA, Freund's complete adjuvant; NN, balanced diet; NNI, balanced diet treated with indomethacin.

*Corresponding author: Professor Dr M. B. de Sousa Maia, fax +55 812126 8976, email maria.maia@pq.cnpq.br 
system $^{(12)}$. It is well known that the development of an uncontrolled inflammatory response has been implicated in the pathogenesis of various chronic diseases such as atherosclerosis and rheumatoid arthritis. Thus, it is clear that nutritional deficiency can seriously impair the repair phase of the inflammatory process, resulting in the critical need for pharmacological intervention in these cases.

Non-steroid anti-inflammatory drugs are the most prescribed medications in the world ${ }^{(13)}$. Indomethacin is a methyl-derived indoleacetic acid pertaining to the non-steroid anti-inflammatory drug class, commonly used to reduce pain, joint stiffness and oedema.

Despite the fact that malnutrition and inflammation may occur concomitantly, nutritional status is not usually investigated as a factor which may affect the pharmacokinetics, and, hence, the pharmacodynamics of drugs.

Thus, it is evident that the understanding of the inflammatory response in individuals subjected to early nutritional deficiency may favour the development of new strategies for predicting disease susceptibility, monitor therapy and ultimately develop new approaches to the prevention and treatment of pathological conditions in which the inflammatory response plays a pivotal role such as rheumatoid arthritis, atherosclerosis and inflammatory bowel disease.

The present study was designed to determine what effect that undernutrition induced by feeding a basic regional diet (rich in carbohydrates and deficient in proteins, lipids, vitamins and minerals) such as is available in rural areas of Pernambuco State, Brazil, has on the inflammatory response exhibited by the adjuvant-induced arthritis in rats, and the anti-inflammatory properties of indomethacin, a standard anti-inflammatory drug

\section{Materials and methods}

\section{Animals}

First-time pregnant Wistar rats (250-300 g) and their offspring (male Wistar rats) from the breeding colony from the Department of Nutrition at the Federal University of Pernambuco (Recife, PE, Brazil) were used. The animals were mated at a ratio of three females to one male in polypropylene cages with dimensions of $430 \times 430 \times 200 \mathrm{~mm}$ in an environment with a temperature of $23 \pm 1^{\circ} \mathrm{C}$ with free access to filtered water. The first day of pregnancy was determined by observing the presence of sperm in the vaginal fluid.

\section{Maternal data and diet}

From the first day of pregnancy, the rats were divided into three groups: (1) a control group (NN) represented by dams that continued on an ad libitum diet of laboratory standard (Labina ${ }^{\circledR}$ Purina do Brasil) composed of $23 \%$ protein, $74 \cdot 4 \%$ carbohydrates and $2.5 \%$ fat; (2) dams that received a basic regional diet (BRD) available in a rural area of Pernambuco State, Brazil, until to term (BRD-g); (3) dams that continued on the BRD throughout the $21 \mathrm{~d}$ lactation period (BRD-gl). The BRD was rich in carbohydrates (82.3\%) and deficient in proteins (10.4\%), lipids (1.86\%), vitamins and minerals, including $\mathrm{NaCl}^{(14)}$. Immediately after birth or weaning, the pups were fed ad libitum with a standard laboratory diet. At day 60 of life, adult male offspring were then subjected to biological assays. The weights of the offspring were measured at birth, 60 and $88 \mathrm{~d}$ of life.

\section{Experimental protocol}

We carried out two experiments.

Experiment $A$. To assess the impact of early malnutrition on the severity of the chronic inflammatory response of adult offspring, experimental arthritis was induced by inoculation of $0.2 \mathrm{ml}$ of a commercially prepared Freund's complete adjuvant (FCA; Sigma Aldrich, St Louis, MO, USA) into the right hind paw ${ }^{(15)}$ of adult male offspring. The animals were allowed to develop chronic inflammation over a period of $28 \mathrm{~d}$ and divided into three groups ( $n$ ) according to their respective dams, namely: (1) NN, (2) BRD-g and (3) BRD-gl. Since they would be used as a control to experiment $\mathrm{B}$, all animals received a daily dose (via oral administration) of $0.9 \%$ saline $(5 \mathrm{ml} / \mathrm{kg})$ once a day during twenty-eight consecutive days. The volume of hind paw swelling $(\mathrm{ml})$ was measured with an electronic water plethysmometer (Ugo Basile, Comerio, Italy) on days 0 (before), 7, 14, 21 and $28 \mathrm{~d}$ after FCA injection. The severity of hind paw inflammation was calculated by paw volume variation $(\Delta V)$ between each time interval and initial hind paw volume $(t=0)$. The hind paw volume (severity of inflammatory response) in the BRD-g and BRD-gl groups was compared with that in the NN group. At the end of the experiments, blood samples for determination of biochemical (plasma albumin and C-reactive protein (CRP) levels) and haematological variables (total and differential leucocytes) were obtained through the orbital plexus. Counting of total leucocytes was performed in a Coulter TKS cell haematological analyser. Blood smears, stained with May-Grunwald-Giemsa, were used for differential counts of leucocytes (segmented, eosinophils, lymphocytes and monocytes). The tests were performed at the Central Laboratory of the Clinical Hospital, Federal University of Pernambuco. Then, all animals were euthanised in a $\mathrm{CO}_{2}$ chamber.

Experiment $B$. To evaluate the impact of undernutrition on the anti-inflammatory effect of indomethacin in adult offspring, another set of animals was subjected to the same procedure as described above, but instead of saline, they received a daily dose (via oral administration) of indomethacin $(2 \mathrm{mg} / \mathrm{kg})$ during twenty-eight consecutive days. According to their respective dams, they were divided into three groups ( $n$ 6): (1) NNI - that represents nourished adult offspring treated with indomethacin; (2) BRDI-g - that represents undernourished adult offspring treated with indomethacin during gestation; (3) BRDI-gl - that represents undernourished adult offspring treated with indomethacin during gestation and lactation. The anti-inflammatory effect of indomethacin (expressed as a percentage of oedema inhibition) in each group was determined by comparing the hind paw volume between the NNI, BRDI-g and BRDI-gl groups with their respective control groups, NN, BRD-g 
and BRD-gl, according to the formula:

$$
\text { Percentage inhibition }(\% I)=1-V_{\mathrm{t}} / V_{\mathrm{c}} \times 100,
$$

where $V_{\mathrm{t}}$ and $V_{\mathrm{c}}$ represent the average of the differences resulting from the hind paw volume in the group treated with indomethacin and control (untreated), respectively.

\section{Bioethical considerations}

The procedures in the handling of animals followed the recommendations of the Brazilian College of Animal Experimentation (BRAZIL, 1979). The experimental protocol and all procedures of the project were reviewed and approved by the Ethics Committee on Animal Experimentation of the Federal University of Pernambuco (protocol no.: 230760070027/2008-19).

\section{Statistical analysis}

Results are expressed as means and standard deviations. Data were stored in databases in Excel software (Microsoft Office ${ }^{\circledR}$ 2007) and Bioestat ${ }^{\circledR}$ (version 5.0) (both programs were freely obtained by Mamirauá Institute). The normality of the data was checked by the Shapiro-Wilk test; quantitative variables were analysed according to descriptive statistics to determine means and standard deviations. When necessary, Student's $t$ test for dependent samples was employed to compare the means of the control and experimental groups, setting $P<0.05$ as the significance threshold for the data.

\section{Results}

Parameters of control and undernourished Wistar rat offspring

As detailed in Table 1, maternal undernutrition impaired fetal growth, verified by low birth weights, as well as a reduction in the number of offspring born to the undernourished dams compared with the nourished ones (NN).

\section{Biochemical and haematological parameters of control and undernourished adult male Wistar rat offspring}

Plasma levels of albumin and CRP in the NN, BRD-g and BRD-gl groups were statistically different. During inflammation, plasma levels of CRP significantly increased, while those of albumin were lower in all the groups in relation to noninflamed animals. However, plasma levels of albumin and CRP were lower in the undernourished groups (BRD-g and BRD-gl) when compared with animals from the control group (NN). With regard to total and differential counts of leucocytes, these parameters did not differ among the three groups (Table 2).

\section{Influence of early undernutrition on severity of inflammatory response}

In nourished and undernourished animals, a single injection of FCA $(0.2 \mathrm{ml})$ in the right paw of each rat produced unilateral swelling of the paw due to oedema over a period of $28 \mathrm{~d}$. However, the chronic inflammatory response induced by FCA in undernourished rats differed markedly from that evoked in matching controls (NN). Fig. 1 shows the development of FCA-induced arthritic lesion. A comparatively lower $(P<0.05)$ magnitude of swelling in the hind paw (severity of the inflammatory response) was observed in the BRD-g and BRD-gl groups compared with that in the NN group up to $14 \mathrm{~d}$ after FCA injection. From the 21st day after the injection of FCA, no difference was observed between the hind paw volumes exhibited by the BRD-g and control groups, whereas in the BRD-gl group, the hind paw volumes remained significantly lower $(P<0.05)$ than those observed in the NN group. It is also interesting to note that in the NN group, hind paw volume $(\mathrm{ml})$ began to increase on day 7 , with a peak on day 14 and thereupon it decreased progressively, whereas in the BRD-g and BRD-gl groups, the peaks were on day 21 and remained elevated until the end of the experiment.

\section{Influence of undernutrition on the anti-inflammatory effect of indomethacin}

Fig. 2 shows the evolution of hind paw swelling of the nourished and undernourished groups treated with indomethacin $(2 \mathrm{mg} / \mathrm{kg})$. As found for the chronic inflammatory response,

Table 1. Characteristics of Wistar rat offspring of nourished (NN) and undernourished dams that received a basic regional diet (BRD) during gestation (BRD-g) or throughout the gestation and lactation periods (BRD-gl)

(Mean values and standard deviations, ten animals per group)

\begin{tabular}{|c|c|c|c|c|c|c|}
\hline \multirow[b]{2}{*}{ Groups } & \multicolumn{2}{|c|}{ Control, NN } & \multicolumn{2}{|c|}{ BRD-g } & \multicolumn{2}{|c|}{ BRD-gl } \\
\hline & Mean & SD & Mean & SD & Mean & SD \\
\hline Number of fetuses & $11 \cdot 2$ & 0.83 & $8 \cdot 8^{*}$ & 0.83 & $8 \cdot 8^{*}$ & 0.83 \\
\hline \multicolumn{7}{|l|}{ Weight of pups (g) } \\
\hline At birth & $6 \cdot 23$ & 0.27 & $4 \cdot 15^{*}$ & 0.15 & $4 \cdot 5^{*}$ & 0.15 \\
\hline At day 22 (weaning) & $50 \cdot 43$ & 1.48 & $43 \cdot 42^{*}$ & $3 \cdot 29$ & $25 \cdot 5^{\star}$ & $7 \cdot 7$ \\
\hline At day 60 & 240 & $36 \cdot 9$ & $238 \cdot 1$ & 21.06 & $186 \cdot 8^{\star}$ & 24 \\
\hline At day 88 & 293.4 & 38 & $282 \cdot 2$ & $30 \cdot 9$ & 263 & $40 \cdot 5$ \\
\hline
\end{tabular}

* Mean value was significantly different from that of the control group (NN) $(P<0.05$.) 
Table 2. Total and differential leucocyte counts and blood levels of albumin and C-reactive protein (CRP) of nourished (NN) and undernourished adult male Wistar rat offspring (day 88 of life) from dams that received a basic regional diet (BRD) during the gestation (BDR-g) or throughout the gestation and lactation periods (BDR-gl)

(Mean values and standard deviations, five animals per group)

\begin{tabular}{|c|c|c|c|c|c|c|}
\hline & \multicolumn{2}{|c|}{$\mathrm{NN}$} & \multicolumn{2}{|c|}{ BRD-g } & \multicolumn{2}{|c|}{ BRD-gl } \\
\hline & Mean & SD & Mean & SD & Mean & SD \\
\hline Albumin (g/l) & $43^{\mathrm{a}}(49 \cdot 8)^{*}$ & $1.7(2.3)^{*}$ & $35.4 \dagger$ & $2 \cdot 7$ & $34 \dagger$ & $2 \cdot 3$ \\
\hline CRP $(\mathrm{mg} / \mathrm{l})$ & $117 \cdot 8^{a}(93 \cdot 8)^{*}$ & $3.5(14)^{\star}$ & $110 \cdot 4 \dagger$ & $2 \cdot 2$ & $107 \cdot 8 \dagger$ & $3 \cdot 2$ \\
\hline \multicolumn{7}{|c|}{ Leucocytes (per mm³) } \\
\hline Total & 16740 & 3566.9 & 17625 & 377.4 & 17360 & 901.6 \\
\hline \multicolumn{7}{|l|}{ Differential } \\
\hline Neutrophil & 3051.4 & $1274 \cdot 8$ & 2258 & $101 \cdot 8$ & 1929 & 1103 \\
\hline Eosinophil & 251 & 178.6 & 141 & 39.4 & 128 & 33.8 \\
\hline Basophil & $870 \cdot 4$ & 592.9 & 872 & 628.6 & 821 & 603.9 \\
\hline Lymphocyte & $12520 \cdot 8$ & 3099 & $14262 \cdot 7$ & 1333.4 & 14284 & $952 \cdot 8$ \\
\hline Monocyte & $44 \cdot 2$ & $60 \cdot 6$ & 88.2 & 90 & $97 \cdot 3$ & $82 \cdot 9$ \\
\hline
\end{tabular}

* The values within parentheses refer to non-inflamed animals.

$\uparrow$ Mean value was significantly different from that of the control group (NN) $(P<0.05)$

the pharmacological response to indomethacin in undernourished animals also differed markedly from that observed in the matching controls. Although treatment with indomethacin attenuated hind paw oedema in all the groups studied (NNI, BRDI-g and BRDI-gl), its efficacy as an anti-inflammatory agent was lower $(P<0.05)$ in the BRDI-g and BRDI-gl groups compared with that in the NNI group. Moreover, this effect can be observed from the 7th day of treatment in NNI animals (Fig. 2(a)), whereas in undernourished animals, it was only evident from the 14th and 21st day for the BRDI-g (Fig. 2(b)) and BRDI-gl groups (Fig. 2(c)), respectively

\section{Discussion}

In the present study, a monoarthritic model induced by Freund's adjuvant was used to investigate the impact of early undernutrition on the chronic inflammatory response and the pharmacological response to indomethacin in adult

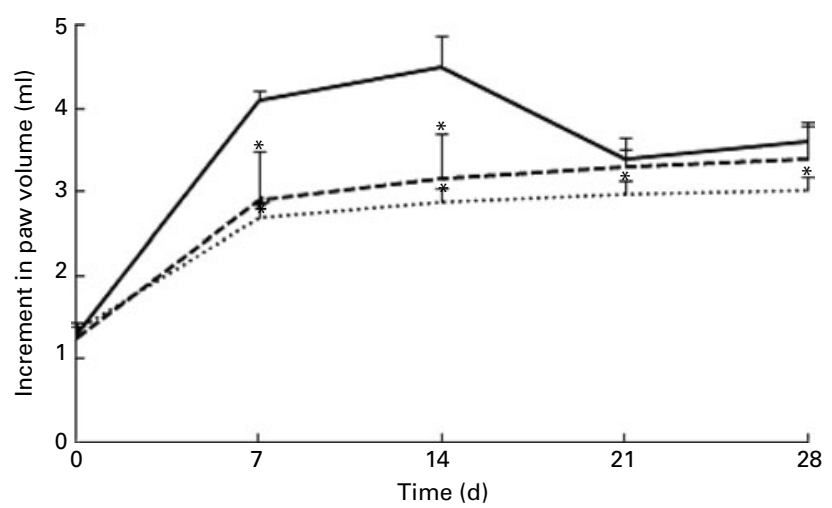

Fig. 1. Time course of hind paw swelling on days 0 (before), 7, 14, 21 and $28 \mathrm{~d}$ after injection with Freund's complete adjuvant $(0.2 \mathrm{ml})$ in nourished $(\mathrm{NN},-)$ and undernourished adult male rat offspring that experienced nutritional deficits during the gestation (basic regional diet during gestation, ---) or throughout the gestation and lactation periods (basic regional diet during gestation and lactation, ........). Values are means (five animals per group), with standard deviations represented by vertical bars. * Mean value was significantly different from the control group (NN) $(P<0.05)$. rat offspring undernourished during gestation or during gestation and lactation.

There is good evidence that maternal food restriction predisposes the offspring to many chronic diseases in its adult life, including renal ${ }^{(16)}$ and cardiac $^{(17)}$ diseases, arterial hypertension $^{(2)}$, diabetes ${ }^{(18)}$, hypothalamic-pituitary-adrenal axis function alterations ${ }^{(19)}$ and leucocyte reduction for the inflammatory site ${ }^{(5)}$. Additionally, it has also been illustrated that newborns of dams subjected to intrauterine undernutrition have consistently lower body weights $(1,8,16,19,20)$ and litter size at birth ${ }^{(1,18)}$ compared with the nourished ones.

The results of the present study show that BRD-g and BRD-gl rats presented clear symptoms of undernutrition (Table 1) and corroborated the above-mentioned observations.

It is well established that the inflammatory response is an unspecific defence mechanism of the body, which may be activated by any factors that predict tissue damage, occurring in three distinct phases: (1) transitory acute phase, characterised by local vessel dilatation and increased capillary permeability, which results in the formation of a local inflammatory exudate; (2) late subacute phase, characterised by the infiltration of white and phagocytary cells; (3) chronic phase, in which fibrosis and tissue degeneration occur. In a healthy individual, this coordinated set of physiological actions results in a competent inflammatory response that serves to fight against infection and reduce tissue injury, and thus, to promote recovery from external stressors before the involvement of the specific immune system ${ }^{(10,12)}$.

With regard to intensity of the chronic inflammatory response, the nourished and undernourished groups showed a significant difference. A comparatively lower $(P<0.05)$ magnitude of hind paw swelling was produced after FCA injection in adult offspring from undernourished dams (BRD-g and BRD-gl) compared with the nourished ones (NN), suggesting a decrease in the effectiveness of the inflammatory response. As can be verified, the resultant hind paw swelling developed more slowly and was not as marked as in NN animals, and the extent of the inflammatory response was lower (Fig. 1) the longer the offspring were exposed to nutritional deficits. However, the 

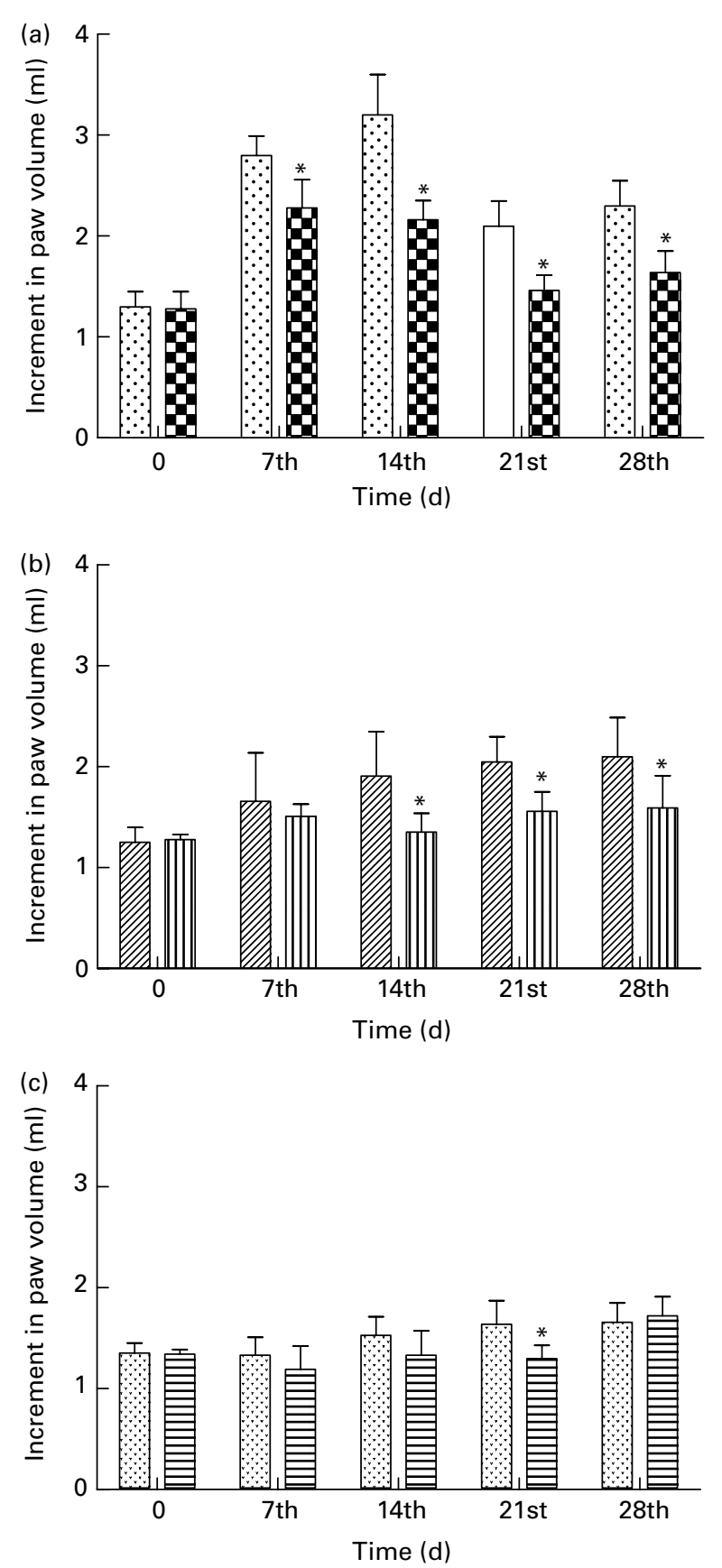

Fig. 2. Effect of oral treatment with indomethacin $(2 \mathrm{mg} / \mathrm{kg})$ on hind paw oedema of nourished and undernourished adult male Wistar rat offspring. Values are means (five animals per group), with standard deviations represented by vertical bars. * Mean values were significantly different from that of the corresponding control group (balanced diet $(\mathbf{0}) \mathrm{v}$. balanced diet treated with indomethacin ( $\mathbf{\square})$; basic regional diet during gestation $(\boldsymbol{\nabla}) \boldsymbol{v}$. basic regional diet treated with indomethacin during gestation $(\mathbb{m})$; basic regional diet during gestation and lactation $(\Xi) \mathrm{v}$. basic regional diet treated with indomethacin during gestation and lactation $($ 目) $)(P<0.05)$.

impact of intra-uterine malnutrition on the inflammatory response of adult offspring does not seem to depend on the stage of pregnancy at which it is introduced. Experimental findings have shown that offspring exposed to a nutrient-deprived environment from $10 \mathrm{~d}$ of pregnancy also demonstrate an attenuated inflammatory response ${ }^{(8)}$.
Previous studies of Barja-Fidalgo et al. ${ }^{(21)}$ show that animals subjected to malnutrition presented a less intense inflammatory response when induced by carrageenin, supposedly as a consequence of reduced leucocyte adhesion to the vascular endothelium with consequent perivascular migration and lower oedema formation. Landgraf et $a l .{ }^{(7)}$ reported that adult offspring that were subjected to intra-uterine undernutrition presented a significant reduction in leukotriene- $\mathrm{B}_{4}$ production, which is of considerable importance in the process of leucocyte chemotaxis, after stimulation with TNF- $\alpha$. It is known that an effective leucocyte margination, endothelium aggregation, endothelial transmigration, as well as an effective recruitment of blood leucocytes, to the inflammatory site are essential steps for an effective inflammatory response. Important parameters associated with leucocyte migration are related to the availability of circulating leucocytes as well as the regulation of migration of these cells from the blood, which is facilitated by the interaction of adhesive molecules with the vascular endothelium. Our data reveal that there were no statistically significant differences in leucocyte counts between the nourished and undernourished animals. Thus, the low-level inflammatory response found in the undernourished groups cannot be attributed to the circulating leucocytes. In contrast to the present findings, Landgraf et al. ${ }^{(7)}$ found a reduction in leucocyte count, but they also showed that intra-uterine undernutrition caused functional deficits in adhesion molecules (reduced L-selectin expressions) and decreased leucocyte migration to the inflammatory site in adult rat offspring. Other studies have shown that undernutrition predisposes subjects to a low-grade local ${ }^{(6)}$ and systemic inflammatory response ${ }^{(9)}$. The difference between their results and the one obtained in the present study may perhaps be explained by the difference between the dietary rations used. Despite this conflict, taken as a whole, the collective evidence suggests that prenatal and/ or postnatal nutritional deficits may impair the capacity of the inflammatory response to noxious stimuli in adult offspring.

Although, in principle, prenatal and/or postnatal undernutrition seems advantageous, since undernourished animals showed a lower inflammatory response (Fig. 1), a more detailed analysis of this result suggests that if the experiment had been extended beyond $28 \mathrm{~d}$, the inflammatory response, while still smaller in magnitude, may have been found to be much longer in duration. According to Bistrian ${ }^{(10)}$, lowgrade, persistent, chronic inflammation, as can be observed in obesity, diabetes and the metabolic syndrome, can lead to serious health risks.

CRP and albumin are important blood markers of the acute phase of the inflammatory response. They play important roles in the modulation of the rate of structural protein synthesis, hormonal transport, local modulation of hormonal effects, neutralisation of the potentially toxic products of the inflammatory response, inhibition of microbial invasion and localisation of bacteria ${ }^{(12)}$. Our data reveal that the albumin and CRP levels were statistically reduced $(P<0.05)$ in the BRD-g and BRD-gl groups compared with those in the NN group, discordant from those found by Desai et al. ${ }^{(8)}$, who detected higher levels of CRP in the offspring undernourished during pregnancy or lactation compared with the control 
group. One must consider that the age of animals, as well as the type of dietary manipulation applied to the studies, if they are different, can be decisive for this disagreement. Once the blood samples for analysis were taken at $88 \mathrm{~d}$ of life and when no differences in weight gain between groups were found, we excluded the nutritional state as a likely explanation for this reduction. Acute-phase proteins are synthesised primarily in the liver from the stimulation of IL- 6 secreted during the inflammatory events mainly by monocytes and macrophages ${ }^{(22)}$; however, when we compared differential leucocyte counts, we found no differences between the BRD-g, BRD-gl and NN groups. It is known that in many mammals subjected to intrauterine malnutrition, there is a redistribution of blood flow in utero to protect important tissues, especially the brain. This adaptation can lead to important costs for tissues other than the brain, notably the liver and other abdominal viscera ${ }^{(2)}$. Thus, other factors such as decreased IL-6 production provoked by impaired hepatic function developed during the gestation and/or the gestation and lactation periods should not be discarded. Histopathological studies of liver tissue and levels of IL-6 and other interleukins are being developed in our laboratory in order to test this hypothesis.

Once it was established that early undernutrition attenuated the chronic inflammatory response, we also verified its influence on the therapeutic efficacy of indomethacin, a standard anti-inflammatory treatment, against adjuvant-induced arthritis over a sustained time period of $28 \mathrm{~d}$.

Non-steroidal anti-inflammatory drugs (e.g. indomethacin, ibuprofen and naproxen) are distinguished as a class by the high degree to which they bind to plasma protein. Indomethacin is a weak organic acid (1-(4-chlorobenzoyl)-5-methoxy-2methyl-1- $H$-indole-3-acetic acid), which binds preferentially to albumin (90-99\%), and undergoes hepatic metabolism and renal excretion.

In addition to the chronic inflammatory response, undernutrition can also affect the pharmacological efficacy of indomethacin. Long-term treatment (over a period of $28 \mathrm{~d}$ ) with indomethacin resulted in a significantly lower $(P<0.05)$ reduction in hind paw swelling in the BRDI-g (Fig. 2(b)) and BRDI-gl (Fig. 2(c)) groups compared with the control group (Fig. 1). It is interesting to note that the longer the offspring were exposed to nutritional deficits, the lower the pharmacological efficacy of indomethacin therapy was.

In the present study, we have shown that plasma albumin was significantly reduced in the undernourished groups. It is known that only the free drug (unbound to plasma proteins) crosses the endothelium of blood vessels and interacts with their pharmacological target. Recognising that if there is a reduction in plasma albumin concentration, there are higher concentrations of unbound indomethacin in the bloodstream, it is reasonable to expect a higher anti-inflammatory effect in undernourished animals. However, in contrast to that expected, the anti-inflammatory effect was lower in the undernourished groups compared with the well-nourished group. Although the objective of the present study was not to investigate the pharmacokinetics of indomethacin, some explanations can be used for this result. It is known that between 10 and $20 \%$ of indomethacin is excreted unchanged in the urine. The amount of drug entering the tubular lumen by filtration depends on the glomerular filtration rate and the extent of plasma binding of the drug; only the unbound drug is filtered. Since the half-life of a drug is inversely proportional to its rate of elimination, an increase in the concentration of unbound indomethacin in malnourished groups may accelerate renal clearance, leading to a reduction in its half-life what could justify its lower anti-inflammatory activity in these animals. Furthermore, as discussed earlier, liver and other abdominal viscera could be impaired during intra-uterine undernutrition. In accordance with O'Keefe ${ }^{(23)}$, chronic malnutrition impairs digestive and absorptive function because food and nutrients are not only the major trophic factors to the gut but also provide the building blocks for digestive enzymes and absorptive cells. The lower antiinflammatory activity of indomethacin in undernourished animals may also be attributed to a deficit in the intestinal absorption caused by intra-uterine malnutrition.

At the moment, our data do not allow us to point to a definitive reason to explain the reduced efficacy of indomethacin in the chronic inflammatory response of undernourished adult offspring. The lower anti-inflammatory efficacy of indomethacin may not only be due to pharmacokinetic changes. The possibility of the involvement of pharmacodynamic factors should not be discarded. Deeper insight into these pharmacokinetic considerations awaits further investigation.

\section{Conclusions}

Early undernutrition attenuated the chronic inflammatory response, and the longer the offspring were exposed to nutritional deficits, the lower the extent of the chronic inflammatory response was. Blood levels of the acute-phase proteins (albumin and CRP) are reduced in adult animals subjected to early undernutrition. The leucocyte count (total or differential) was not altered in animals subjected to early undernutrition. Indomethacin showed different efficacy in attenuating the progression of arthritis induced by FCA in the normally nourished and undernourished groups. Its anti-inflammatory effect was less pronounced in undernourished offspring.

\section{Acknowledgements}

The present study was supported by the Graduate Program of Pathology of the Federal University of Pernambuco. None of the authors had any personal or financial conflict of interest. M. B. d. S. M., T. d. O. A., T. G. d. S. and S. R. A. d. M. contributed to the study design, and T. d. O. A., M. B. d. S. M. and E. d. S. F. performed the data collection. M. B. d. S. M., T. d. O. A. and E. d. S. F. were involved in the data analysis and the interpretation of the results. M. B. d. S. M., T. d. O. A. and E. d. S. F. contributed to the writing of the manuscript.

\section{References}

1. Gurmini J, Cecilio WA, Schuler SL, et al. (2005) In-uterus malnutrition and its changes in the small bowel of Wistar rats at birth and after lactation. J Bras Patol Med Lab 41, 271-278. 
2. Barker DJ (2000) In utero programming of cardiovascular disease. Theriogenology 53, 555-574.

3. Eriksson J, Forsen T, Tuomilehto J, et al. (2000) Fetal and childhood growth and hypertension in adult life. Hypertension 36, 790-794.

4. Jones C (2002) Foetal programming and coronary heart disease in later life. Liverpool. Br J Nutr 11, 822-826.

5. Chandra RK, Chandra S \& Ghai OP (1976) Chemotaxis, random mobility, and mobilization of polymorphonuclear leucocytes in malnutrition. J Clin Path 29, 224-227.

6. Leme-brasil MR, Collares EF, Verissimo MS, et al. (1980) Acute inflammatory responses in rats with protein-calorie malnutrition. Agents Actions 10, 445-450.

7. Landgraf MA, Tostes Rde C, Borelli P, et al. (2007) Mechanisms involved in the reduced leukocyte migration in intrauterine undernourishment. Nutrition 23, 145-156.

8. Desai M, Gayle DA, Casillas E, et al. (2009) Early undernutrition attenuates the inflammatory response in adult rat offspring. J Matern Fetal Neonatal Med 22, 571-575.

9. Deitch EA, Ma WJ, Ma L, et al. (1990) Protein malnutrition predisposes to inflammatory-induced gut-origin septic states. Ann Surg 211, 560-567.

10. Bistrian B (2007) Systemic response to inflammation. Nutr Rev 65, 170-172. (Review).

11. Ayala A, Chung CS, Grutkoski OS, et al. (2003) Mechanism of immune resolution. Crit Care Med 31, Suppl., 558-571.

12. Wan JM, Haw MP, Blackburn GL, et al. (1989) Nutrition, immune function, and inflammation: an overview. Proc Nutr Soc 48, 315-335.

13. Rocha RJ, Parrado MJ, Rojas IM, et al. (2007) Intestinal damage by non-steroidal anti-inflammatory drugs. Rev Gastroenterol Mex 72, 43-46.
14. Teodósio NR, Lago ES, Romani SA, et al. (1990) A regional basic diet from Northeast Brazil as a dietary model of experimental malnutrition. Arch Latinoam Nutr $\mathbf{4 0}$, 533-547.

15. Selye H (1949) Further studies concerning the participation of the adrenal cortex in the pathogenesis of arthritis. $\mathrm{BrJ}$ Nutr 2, 1129-1135.

16. Franco MC, Ponzio BF, Gomes GN, et al. (2009) Micronutrient prenatal supplementation prevents the development of hypertension and vascular endothelial damage induced by intrauterine malnutrition. Life Sci 12, 327-333.

17. Roseboom TJ, Van-der-Meulen JHP, Osmond C, et al. (2000) Coronary heart disease after prenatal exposure to the Dutch famine. Heart 84, 595-598.

18. Philips DI, Hirst S, Clark PM, et al. (1994) Fetal growth and insulin secretion in adult life. Diabetologia 37, 592-596.

19. Lesage J, Hahn D, Léonhardt M, et al. (2002) Maternal undernutrition during late gestation-induced intrauterine growth restriction in the rat is associated with impaired placental GLUT3 expression, but does not correlate with endogenous corticosterone levels. J Endocrinol 174, 37-43.

20. Wells JC (2007) Flaws in the theory of predictive adaptive response. Trends Endocrinol Metab 18, 331-337.

21. Barja-Fidalgo C, Souza EPG, Silva SV, et al. (2003) Impairment of inflammatory response in adult rats submitted to maternal undernutrition during early lactation: role of insulin and glucocorticoid. Inflamm Res 52, 470-476.

22. Stenvinkel P, Heimburger O, Paultre F, et al. (1999) Strong association between malnutrition, inflammation, and atherosclerosis in chronic renal failure. Kidney Int 55, 1899-1911.

23. O'Keefe SJ (1996) Nutrition and gastrointestinal disease. Scand J Gastroenterol 220, Suppl., 52-59. 